\section{Underdetection of Ventricular Fibrillation by Wearable Cardioverter-Defibrillator Leading to Sudden Death}

Hiroto Kokubun, MD; Yasushi Miyauchi, MD; Shin Inada, PhD; Wataru Shimizu, MD

A 51-year-old man with end-stage renal failure collapsed at a hemodialysis facility and was transferred after ventricular fibrillation (VF) was successfully terminated by an automated external defibrillator. Coronary angiography revealed $90 \%$ stenosis in the proximal portion of the circumflex artery, where a drug-eluting stent had been deployed. Transthoracic echocardiography revealed severe stenosis of the aortic valve. Insertion of an implantable cardioverter-defibrillator and aortic valve replacement were scheduled for 1 month later, and he was discharged on the 10th hospital day with a wearable cardioverter-defibrillator (WCD) (Life Vest ${ }^{\circledR}$, Zoll Medical Corp., Chelmsford, MA, USA). However, 13 days later, he was found dead.

The WCD recordings were reviewed. At $1 \mathrm{~h}$ before he was found dead, VF started (Figure B) and was accordingly detected 20s later (Figure C). However, as the voltage of VF decreased, the algorithm determined $67 \mathrm{~s}$ later that VF had stopped (Figure D), despite continuation of VF. Figure E shows the same recording as in Figure D, with detectors. The upper gray line demonstrates the lack of morphology match with the baseline recording, which is determined by vectorcardiography, showing that VF detection was stopped because the morphology matched the sinus rhythm over several seconds (Figure E, arrow). Vectorcardiography demonstrated a similarity between baseline sinus rhythm (Figure A) and VF (Figure D). Without any shocks delivered, the rhythm changed to an agonal rhythm 10 min later (Figure F), and eventually he died.

This case demonstrates that a WCD may miss VF, causing sudden death. Incidental vectorcardiographic similarity between sinus rhythm and VF may cause underdetection of VF.

\section{Disclosure}

W.S. is a member of Circulation Journal's Editorial Team.

Received September 17, 2020; revised manuscript received September 18, 2020; accepted September 23, 2020; J-STAGE Advance Publication released online November 17, 2020 Time for primary review: 1 day

Department of Cardiovascular Medicine, Nippon Medical School Chiba-Hokusoh Hospital, Inzai (H.K., Y.M.); Department of Medical Engineering, Faculty of Health Sciences, Morinomiya University of Medical Sciences, Osaka (S.I.); and Department of Cardiovascular Medicine, Nippon Medical School, Tokyo (W.S.), Japan

Mailing address: Yasushi Miyauchi, MD, Department of Cardiovascular Medicine, Nippon Medical School Chiba-Hokusoh Hospital, 1715 Kamagari, Inzai, Chiba 270-1694, Japan. E-mail: miyauchi@, nms.ac.jp

All rights are reserved to the Japanese Circulation Society. For permissions, please e-mail: cj@j-circ.or.jp

ISSN-1346-9843
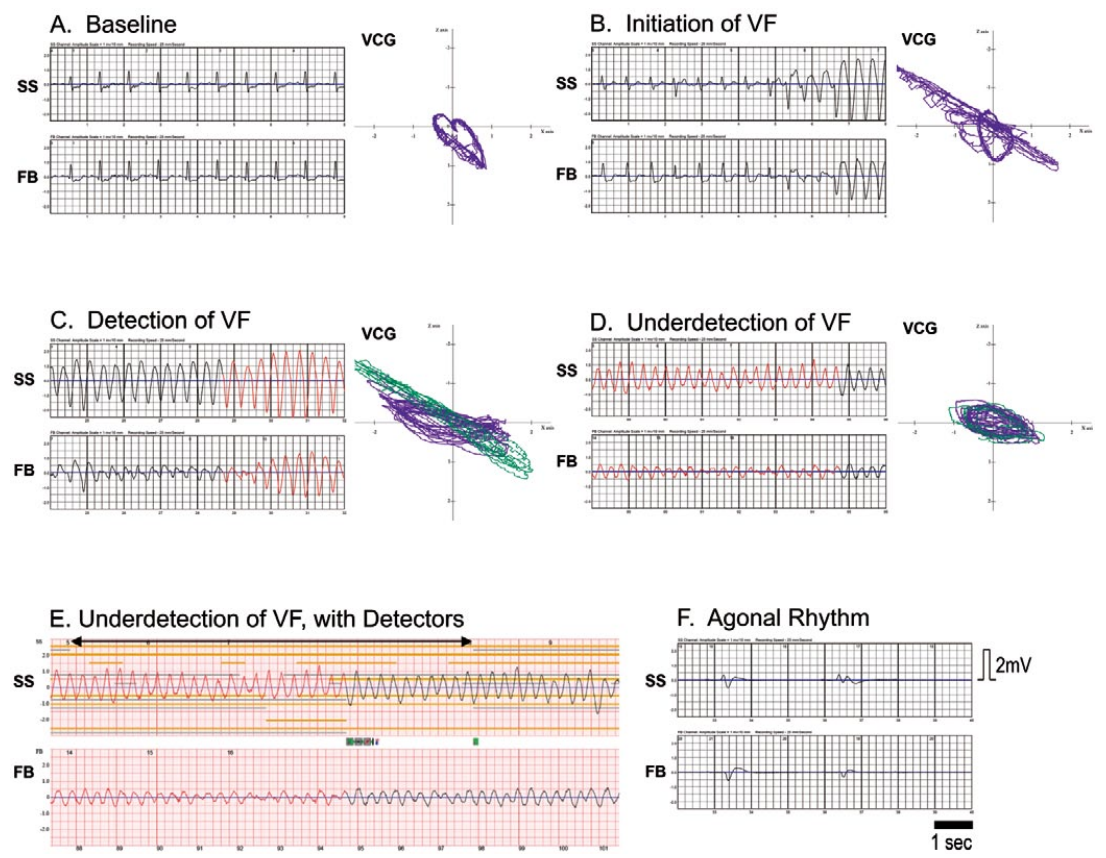

Figure. Side-side (SS) and front-back (FB) electrograms and corresponding vectorcardiography (VCG) during baseline (A), initiation (B), detection (C), and underdetection (D) of ventricular fibrillation (VF). (E) Same recordings as in (D), with detectors. (F) Electrograms during agonal rhythm. 